Evolution in Natur und Kultur 
Gerhard Schurz

\section{Evolution in Natur und Kultur}

Eine Einführung in die verallgemeinerte Evolutionstheorie 
Gerhard Schurz

Heinrich-Heine-Universität

Institut für Philosophie

Universitätsstraße 1

40225 Düsseldorf

\section{Wichtiger Hinweis für den Benutzer}

Der Verlag, der Herausgeber und die Autoren haben alle Sorgfalt walten lassen, um vollständige und akkurate Informationen in diesem Buch zu publizieren. Der Verlag übernimmt weder Garantie noch die juristische Verantwortung oder irgendeine Haftung für die Nutzung dieser Informationen, für deren Wirtschaftlichkeit oder fehlerfreie Funktion für einen bestimmten Zweck. Der Verlag übernimmt keine Gewähr dafür, dass die beschriebenen Verfahren, Programme usw. frei von Schutzrechten Dritter sind. Die Wiedergabe von Gebrauchsnamen, Handelsnamen, Warenbezeichnungen usw. in diesem Buch berechtigt auch ohne besondere Kennzeichnung nicht zu der Annahme, dass solche Namen im Sinne der Warenzeichen- und Markenschutz-Gesetzgebung als frei zu betrachten wären und daher von jedermann benutzt werden dürften. Der Verlag hat sich bemüht, sämtliche Rechteinhaber von Abbildungen zu ermitteln. Sollte dem Verlag gegenüber dennoch der Nachweis der Rechtsinhaberschaft geführt werden, wird das branchenübliche Honorar gezahlt.

\section{Bibliografische Information der Deutschen Nationalbibliothek}

Die Deutsche Nationalbibliothek verzeichnet diese Publikation in der Deutschen Nationalbibliografie; detaillierte bibliografische Daten sind im Internet über http://dnb.d-nb.de abrufbar.

Springer ist ein Unternehmen von Springer Science+Business Media springer.de

(C) Spektrum Akademischer Verlag Heidelberg 2011

Spektrum Akademischer Verlag ist ein Imprint von Springer
$\begin{array}{lllll}11 & 12 & 13 & 14 & 15\end{array}$
$\begin{array}{lllll}5 & 4 & 3 & 2 & 1\end{array}$

Das Werk einschließlich aller seiner Teile ist urheberrechtlich geschützt. Jede Verwertung außerhalb der engen Grenzen des Urheberrechtsgesetzes ist ohne Zustimmung des Verlages unzulässig und strafbar. Das gilt insbesondere für Vervielfälttigungen, Übersetzungen, Mikroverfilmungen und die Einspeicherung und Verarbeitung in elektronischen Systemen.

Planung und Lektorat: Dr. Andreas Rüdinger, Heidemarie Wolter

Redaktion: Regine Zimmerschied

Satz: klartext, Heidelberg

Umschlaggestaltung: wsp design Werbeagentur GmbH, Heidelberg

Titelfotografie: 10.000 Meisterwerke, The Yorck Projekt: Hieronymus Bosch, Der Garten der Lüste (Detail) 


\section{Inhaltsverzeichnis}

Vorwort und Einleitung . . . . . . . . . . . . . . . . XIII

\section{Teil I}

Woher kommen wir? Evolution in der Natur

1. Von der Genesis zur Evolution: Die historische Ablösung der Evolutionstheorie aus metaphysisch-normativen Entwicklungskonzeptionen . . . . . . . . . . 3

1.1 Fernab von Darwin: Entwicklungstheorien des Common Sense . 3

1.2 Der planvolle Schöpfungsakt: Spielarten des Kreationismus . . . 5

1.3 Zielgerichtete Bildungskraft: Teleologie nach Aristoteles . . . . . 10

1.4 Beginnende Naturwissenschaft: Entwicklungstheorien vor Darwin . . . . . . . . . . . . . . . . . . . . . . . 14

1.5 Die Entstehung der Darwin'schen Evolutionstheorie . . . . . . . . 21

2. The Modern Synthesis: Von Darwin bis zur biologischen Evolutionstheorie der Gegenwart . . . . . . . . . . . . . . . 33

2.1 Mendel und die Mechanismen der biologischen Vererbung . . . . 34

2.2 Theoretische und empirische Populationsgenetik . . . . . . . . 40

2.2.1 Mathematisch-theoretische Populationsgenetik . . . . . . . . . . . 41

2.2.2 Empirische („ökologische“ und experimentelle) Populationsgenetik. . . . . 44

2.3 Genetischer Code und epigenetische Steuerung . . . . . . . . . 45

2.4 Zwischen Stagnation und Revolution: Eckdaten biologischer Evolution. . . . . . . . . . . . . . . . . . . . 52

2.5 Kategorisierung des Evolvierenden: Das Problem der natürlichen Klassifikation biologischer Arten und Gattungen . . . . . . . . 61

3. Was man strengen Kreationisten entgegenhält: Evidenzen für die Evolution . . . . . . . . . . . . . . . . 71

3.1 Direkter empirischer und praktischer Nachweis der evolutionären Selektion 
3.2 Biogeografie: Evolutionäre Erklärung geografischer Variation . . 72

3.3 Unabhängige Methoden der Altersbestimmung . . . . . . . . 75

3.3.1 Fossilfunde, Serien und Lücken . . . . . . . . . . . . . . . . . . 75

3.3.2 Altersbestimmung durch radioaktive Isotope . . . . . . . . . . . . . 77

3.3.3 Altersbestimmung durch neutrale Mutationsraten . . . . . . . . . . 78

3.4 Unabhängige Methoden der Generierung evolutionärer Stammbäume . . . . . . . . . . . . . . . . . . . 79

3.5 Die Bestätigungskraft unabhängiger Evidenzen. . . . . . . . . . 86

4. Was man liberalen Kreationisten und Teleologen entgegenhält: Evidenzen gegen das Designargument und Auflösung von Denkschwierigkeiten . . . . . . . . . . 89

4.1 Suboptimalitäten in der Evolution: Evidenzen gegen das Designargument . . . . . . . . . . . . . . . . . . . . 89

4.2 Kann das denn alles wirklich ohne das Wirken höherer Kräfte entstanden sein? Fundamentale Denkschwierigkeiten und ihre Auflösung . . . . . . . . . . . . . . . . . 99 94

4.2.1 Zur Wahrscheinlichkeit der Entstehung höheren Lebens . . . . . . . . . . 94

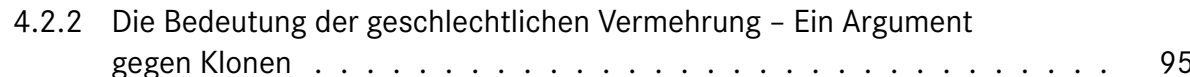

4.2.3 Das Problem der Makrotransformationen: Exadaption und Präadaption . 98

4.3 Aber wie entsteht die erste Zelle? Kooperation unter RNSMolekülen und die präbiotische Evolution primitivsten Lebens . . 101

4.4 Wunder des Lebens und Wunder der Zeit: Quasigöttliche Unvorstellbarkeiten

5. Das anthropische Prinzip: Auferstehung des Kreationismus in der Kosmologie?

5.1 Astrobiologie: Wie (un)wahrscheinlich ist die Entstehung von Leben im Universum? . . . . . . . . . . . . . . . . . . . . . . . 107

5.2 Das anthropische Prinzip: Begründung vs. Erklärung . . . . . . 113

5.3 Alternative Erklärungen des Unwahrscheinlichen:

Vier Zugänge zur Letztfrage .

5.4 In welchem Sinn unwahrscheinlich? Objektive und subjektive Wahrscheinlichkeit . . . . . . . . . . . . . . . . . . 117

5.5 Ist der Erklärungsdurst stillbar? Zur Illusion von Letzterklärungen . 119

5.6 Kreationismus von vornherein unwissenschaftlich? Empirisch kritisierbare vs. unkritisierbare Kreationismusformen . . . . . . . 120

5.7 Rationalisierter Kreationismus und Voraussagekriterium: Das Abgrenzungsproblem . . . . . . . . . . . . . . . . . . . 122

5.8 Bayesianische Bestätigung des Kreationismus? . . . . . . . . . . 124

5.9 Wissenschaftliche Erklärungsversuche: Schwarze Löcher und Urknalle - Evolution des Kosmos? . . . . . . . . . . . . . . . . 


\section{Teil II}

\section{Evolution überall? Verallgemeinerung} der Evolutionstheorie

6. Prinzipien moderner Evolutionstheorie und ihre Verallgemeinerung.

6.1 Die drei Darwin'schen Module: Evolution als rekursiver

Algorithmus . . . . . . . . . . . . . . . . . . . . . 131

6.2 Grundlektionen evolutionären Denkens . . . . . . . . . . . . . 133

6.2 .1 Was wird selektiert? . . . . . . . . . . . . . . . . . . . . . . 133

6.2.2 Subversion von Ziel, Fortschritt und Essenz . . . . . . . . . . . . . . 134

6.2.3 Gerichtete Evolution und Stabilität der Selektionskräfte . . . . . . . . . . 135

6.2.4 Elimination und Produktion von Vielfalt. . . . . . . . . . . . . . . . 136

6.2 .5 Evolution der Evolutionsfähigkeit. . . . . . . . . . . . . . . . . . . 137

6.2.6 Alternativen zum Darwinismus oder Varianten eines vereinheitlichenden Paradigmas? . . . . . . . . . . . . . . . . . . . 138

6.3 Verallgemeinerung der Evolutionstheorie . . . . . . . . . . . . 140

6.3 .1 Kulturelle Evolution . . . . . . . . . . . . . . . . . . . . . . . . . . . 140

6.3 .2 Individuelle Evolution . . . . . . . . . . . . . . . . . . . . . . 141

6.3.3 Verallgemeinerte Evolutionstheorie: Theorie oder Metapher? . . . . . . . 142

6.3.4 Weitere Ebenen der Evolution . . . . . . . . . . . . . . . . . . . 143

6.4 Protoevolution physikochemischer Strukturen auf der Basis von begrenzter Variation und Retention . . . . . . . . . . . . 143

6.5 Die Protoevolution von Planetensystemen. . . . . . . . . . . . . . 145

6.6 Die Verschachtelungshierarchie evolutionärer Systeme: Größenordnungen und Zeitfenster . . . . . . . . . . . . . . . 147

7. Wissenschaftstheoretische Grundlagen der Evolutionstheorie . . . . . . . . . . . . . . . . . . . . . . . 149

7.1 Systemtheoretische Grundlagen: Geschlossene vs. offene Systeme . . . . . . . . . . . . . . . . . . . . . . . . . . . 149

7.2 Selbstregulative Systeme, evolutionäre Systeme und ihre normischen Gesetzmäßigkeiten .

7.3 Stufen der evolutionären Selbstregulation und Selbstorganisation . . . . . . . . . . . . . . . . . . 154

7.4 Evolutionäre Normalität und genuine evolutionäre Funktion . . . 156

7.5 Survival of the Fittest - Eine Tautologie? Gehalt und Überprüfbarkeit der Evolutionstheorie

7.6 Naturgesetze vs. Systemgesetze - Verallgemeinerte Evolutionstheorie als übergreifendes Paradigma „höherer“ Wissenschaften

7.7 Supervenienz ohne Reduzierbarkeit: Zum Verhältnis von Biologie (bzw. „höheren“ Wissenschaften) und Physik . 
8. Konflikte zwischen Evolutionstheorie und humanistischem Weltbild: Die ethische Dimension

8.1 Psychologische und gesellschaftliche Widerstände gegen darwinistische Weltbildumwälzungen

8.2 Konflikte zwischen Evolutionstheorie und humanistischer Moral? . . . . . . . . . . . . . . . . . . . 173

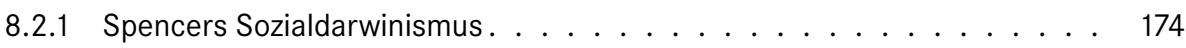

8.2.2 Drei Thesen . . . . . . . . . . . . . . . . . . . . . . . . . . . . . . . . . . . . . . . 177

8.2.3 Rassismus . . . . . . . . . . . . . . . . . . . . . . 177

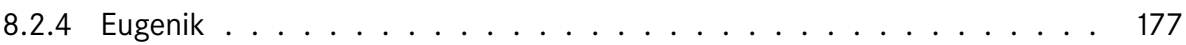

8.3 Brückenprinzipien für eine evolutionäre Ethik . . . . . . . . . . . . 179

8.3.1 Sein und Sollen . . . . . . . . . . . . . . . . . . . . . . . . 179

8.3.2 Gene vs. Umwelt: Hat die Soziobiologie ideologische Funktion? . . . . . . 180

8.3.3 Brückenprinzipien zwischen Sein und Sollen . . . . . . . . . . . . . . . . 181

8.4 Moral aus evolutionärer Sicht: Die Evolution sozialer Kooperation . . . . . . . . . . . . . . . . . . . 183

8.5 Sozialdarwinismus vs. soziale Evolutionstheorie . . . . . . . . 185

\section{Teil III}

Menschlich - Allzu menschlich: Evolution der Kultur . . . . 189

9. Kulturelle Evolution . . . . . . . . . . . . . . . . . . . . . . 191

9.1 Meme: Entstehung und Grundprinzipien der kulturellen Evolutionstheorie . . . . . . . . . . . . . . . . . . 192

9.2 Evidenzen für kulturelle Evolution . . . . . . . . . . . . . . . 197

9.2.1 Nichtreduzierbarkeit der KE auf biologisch-genetische Evolution . . . . . . 198

9.2.2 Unbegrenzte geistig-kulturelle Kreativität und Nichtreduzierbarkeit der KE auf individuelle Einzelleistungen . . . . . . . . . . . . . . . . . 201

9.2.3 Die Nichtintentionalität der KE: Nichtreduzierbarkeit auf Handlungstheorie . . . . . . . . . . . . . . . . 203

9.2.4 Scheitern alternativer Theorien - Kritik soziologistischer Lerntheorien . . . 205

9.2.5 Parallelitäten zwischen BE und KE . . . . . . . . . . . . . . . . . . 206

9.3 Kritik an der Memetik als eigene Wissenschaft . . . . . . . . . . . 208

9.4 Das Problem der Lokalisation und Identität von Memen . . . . . . 210

9.5 Mechanismen der kulturellen Reproduktion . . . . . . . . . . . . 214

9.5.1 Imitation und Lernen. . . . . . . . . . . . . . . . . . . . . . . . . . . . . . . . . . . . 214

9.5.2 Kulturelle Evolution bei Tieren . . . . . . . . . . . . . . . . . 216

9.5.3 Replikation, Reproduktion, Informationsübertragung und Retention . . . . 217

9.5.4 Mischvererbung, Stammlinienvereinigung und kulturelle Quasispezies . . . 222

9.6 Detailfragen der kulturellen Variation . . . . . . . . . . . . . . 225

9.6.1 Gerichtete Variation . . . . . . . . . . . . . . . . . . . 225

9.6.2 Makromutationen .................... . . 226

9.6.3 Hohe Variationsrate . . . . . . . . . . . . . . . . . 226 
9.6.4 Variation als Interpretation: Interpretative Wissenschaften aus evolutionstheoretischer Perspektive . . . . . . . . . . . . . . . . . 227

9.7 Detailprobleme der kulturellen Selektion . . . . . . . . . . . . 229

9.7.1 Fertilitätsfitness und Vitalitätsfitness von Memen . . . . . . . . . . . . . 229

9.7.2 Selektionskriterien und Rationalitätskriterien - Ein Zusammenhang? . . . 231

9.7.3 Fundamentalismus und Aufklärung: Weltanschauungen als Selektionsmechanismen . . . . . . . . . . . . . . . . . 232

9.7.4 Autoselektion: Kopplung von Variation und Selektion . . . . . . . . . . . . 233

9.7.5 Kulturelle Nischenkonstruktion und Umweltinduktion . . . . . . . . . . . 234

9.8 Häufigkeitsabhängige Selektion. . . . . . . . . . . . . . 235

9.8.1 Reflexive Häufigkeitsabhängigkeit . . . . . . . . . . . . . . . . . . 235

9.8.2 Negativ-reflexive Häufigkeitsabhängigkeit und nachhaltige kulturelle Vielfalt. . . . . . . . . . . . . . . . . 236

9.8.3 Interaktive Häufigkeitsabhängigkeit und evolutionäre Spieltheorie . . . . . 236

9.9 Zusammenfassung der Unterschiede der KE gegenüber der BE. . 237

10. Leistungen und exemplarische Anwendungsbereiche der kulturellen Evolutionstheorie . . . . . . . . . . . . . . . 239

10.1 Beispiele für überzogene oder unfaire Kritiken an der KE-Theorie . . . . . . . . . . . . . . . . . . . 239

10.2 Diamonds Menschheitsgeschichte als Musterbeispiel implizit-evolutionärer Geschichtsschreibung . . . . . . . . . 242

10.3 Das Beispiel der technologischen Evolution . . . . . . . . . . . . 249

10.4 Weitere Anwendungen der KE-Theorie im Überblick . . . . . . . . 255

\section{Interaktionen zwischen der kulturellen, biologischen} und individuellen Evolution . . . . . . . . . . . . . . . . . . 257

11.1 Individuelle Evolution und Arten des individuellen Lernens . . . . 257

11.1.1 Prägung . . . . . . . . . . . . . . . . . . . 257

11.1.2 Klassische Konditionierung als Umweltinduktion . . . . . . . . . . . . 258

11.1.3 Operante Konditionierung als Evolutionsprozess . . . . . . . . . . . . . . 259

11.1.4 Einsicht und Konstruktion kognitiver Modelle. . . . . . . . . . . . . . . . 260

11.2 Relative Vorteile von BE, KE und IE . . . . . . . . . . . . . . . . 261

11.3 Interaktionen zwischen KE und IE. . . . . . . . . . . . . . . . 261

11.3.1 Arten sozialen Lernens. . . . . . . . . . . . . . . . . . . . . . . . . . . 261

11.3.2 Transfer von IE zu KE und verallgemeinerter Lamarckismus . . . . . . . . 262

11.4 Interaktionen zwischen KE und BE . . . . . . . . . . . . . 263

11.4.1 Wilson-Effekt und Baldwin-Effekt - Ein Quasi-Lamarckismus . . . . . . . . 263

11.4.2 Gene vs. Kultur: Gegenläufigkeiten zwischen KE und BE . . . . . . . . . . 265

11.5 Ein „heißes Eisen“: Invers-korrelierte Reproduktion am Beispiel des IQ . . . . . . . . . . . . . . . . . . . 267 


\section{Teil IV}

\section{Gedankliche Akrobatik: Mathematische Grundlagen und theoretische Modelle der verallgemeinerten}

12. Mathematische Grundlagen der verallgemeinerten Evolutionstheorie . . . . . . . . . . . . . . . . . . 275

12.1 Dynamische Systeme, Differenz- und Differenzialgleichungen . . 275

12.2 Stabilität, Indifferenz und Instabilität von Trajektorien . . . . . . . 278

12.3 Populationsdynamische Beschreibung evolutionärer Systeme . . 281

12.4 Genotypengleichgewichte ohne Selektion -

Das Hardy-Weinberg-Gesetz. . . . . . . . . . . . . . . . . . . . 283

12.5 Einfache Evolution unter den Kräften der Selektion . . . . . . . . 284

12.5.1 BE: Selektion eines dominanten Allels . . . . . . . . . . . . . . . 284

12.5.2 BE: Selektion eines rezessiven Allels . . . . . . . . . . . . . . . . . 290

12.5.3 KE: Selektion eines kulturellen (ungeschlechtlichen) Reprons . . . . . . . 291

12.5.4 Der allgemeine Fall: Differenz-vs. Differenzialgleichung . . . . . . . . . . 293

\section{Theoretische Modelle I: Gerichtete Evolution} ohne Häufigkeitsabhängigkeit . . . . . . . . . . . . . . . . 295

13.1 Evolution unter den Kräften von Selektion und Mutation . . . . 295

13.1.1 BE: Selektion eines (rück)mutierenden dominanten Allels . . . . . . . . . 295

13.1.2 BE: Selektion eines (rück)mutierenden rezessiven Allels - Eine Bifurkation . 297

13.1.3 KE: Selektion eines (rück)mutierenden vorteilhaften Reprons. . . . . . . . 299

13.2 Arten von Polymorphismus . . . . . . . . . . . . . . . . . . 301

13.2.1 Heterozygotenpolymorphismus in der BE. . . . . . . . . . . . . 301

13.2.2 Multiple Nischen, Speziesvielfalt und Polymorphismus . . . . . . . . . . . 303

13.2.3 Multiple Nischen ohne Segregation führen nicht zu Vielfalt . . . . . . . . . 304

13.2.4 Freie Wahl der optimalen Nische führt zu Speziesvielfalt . . . . . . . . . . 305

13.2.5 Multiple Nischen mit Migration führen zu genuinem Polymorphismus. . . . 306

13.2.6 Der mathematisch allgemeine Fall ungeschlechtlicher Evolution . . . . . . 310

14. Theoretische Modelle II: Evolution mit Häufigkeitsabhängigkeit . . . . . . . . . . . . . . . . . 311

14.1 Reflexive Häufigkeitsabhängigkeit . . . . . . . . . . . . . . . 311

14.1.1 Negative Häufigkeitsabhängigkeit - Wenn Evolution zyklisch wird . . . . . 311

14.1.2 Positive Häufigkeitsabhängigkeit - Wenn Evolution zu Extremen tendiert . . 313

14.1.3 Gemischte Häufigkeitsabhängigkeit und Modezyklen . . . . . . . . . . . . 315

14.2 Ökologische Populationsdynamik I: Logistisches Wachstum . . . 316

14.3 Interaktive Häufigkeitsabhängigkeit und ökologische

Populationsdynamik II . . . . . . . . . . . . . . . . . . . . . 318

14.3.1 Interspezieskonkurrenz . . . . . . . . . . . . . . . . . . . . 318

14.3.2 Räuber-Beute-Dynamik. . . . . . . . . . . . . . . . . . . . . . 321 
15. Theoretische Modelle III: Evolutionäre Spieltheorie . . . . 323

15.1 Grundbegriffe der evolutionären Spieltheorie . . . . . . . . . . . . 323

15.1.1 Klassische Spieltheorie . . . . . . . . . . . . . . . . . . . . . 323

15.1.2 Evolutionäre Spieltheorie. . . . . . . . . . . . . . . . . . . . 326

15.2 Grundtypen symmetrischer Zweierspiele . . . . . . . . . . . . . . 332

15.2.1 Defektion-Kooperation (Gefangenendilemma) und die Paradoxie individueller Nutzenmaximierung. . . . . . . . . . . . . . . . . . . . 332

15.2.2 Kampf-Kompromiss (Habicht-Taube) und das Gleichgewicht von Beharren und Nachgeben . . . . . . . . . . . . . . . . . . . . 335

15.2.3 Koordination und das Problem der Einigung . . . . . . . . . . . . . . . . 337

15.3 Spiele mit vielen Strategien: Sensitivität gegenüber Anfangsund Randbedingungen und chaotische Evolutionsverläufe

\section{Teil V}

\section{Gut und Böse, Wahr und Falsch: Die Evolution} von Moral, Wissen und Glaube .

16. Wie gut ist der Mensch? Die Evolution der Kooperation . . 347

16.1 Kooperation, Egoismus und Altruismus . . . . . . . . . . . . 347

16.2 Gruppenselektion bei ständiger Neuformierung . . . . . . . . . . 349

16.3 Korrelierte Paarung und Absprache: Die Evolution von Signalbedeutung . . . . . . . . . . . . . . . . 352

16.4 Tit for Tat: Reziprozität und das iterierte D-K-Spiel mit Gedächtnis . 355

16.5 Entstehung von Kooperation aus Intraspeziessymbiose . . . . . . 358

16.6 Gemeinschaftliche Sanktion und Belohnung: Die evolutionäre Bedeutung von institutionalisierten Sanktionssystemen . . . . . 360

16.7 Spezifisch menschliche Mechanismen der Kooperationsförderung . . . . . . . . . . . 366

16.7.1 Intentionalverstehen, Mitgefühl und Fairness. . . . . . . . . . . . . 366

16.7.2 Betrugsaufdeckung und konditionales Schließen . . . . . . . . . . 367

16.7.3 Erfolgsabhängige Nachahmung und Nachbarschaftsstrukturen. . . . . . . 369

16.7.4 Die Doppelrolle der Religion: Stabilisator von Gruppenidentität und Legitimator von Gruppenkonflikten. . . . . . . . . . . . . . . . . 372

16.7.5 Gewaltenteilung und moderne Demokratien . . . . . . . . . . . 373

17. Wie vernünftig ist der Mensch? Zur Evolution von Kognition und Weltanschauung . . . . . . . . . . . . 375

17.1 Sprachevolution und linguistische Abstammungsbäume . . . . 375

17.2 Sprache und soziale Kognition, Intention und Kausalität: Zur kognitiven Differenz von Schimpanse und Mensch . . . . . . . . 380

17.3 Zwischen Modularität und Universalität: Die evolutionäre Architektur menschlicher Kognition 
17.4 Egozentrischer Bias und verallgemeinerter Placeboeffekt:

Die Grenzen der evolutionären Erkenntnistheorie . . . . . . . . . 390

17.5 Evolution von Religion . . . . . . . . . . . . . . . . . . . . . . 394

17.5.1 Multiple Beibehaltungsgründe für Religion: Der verallgemeinerte

Placeboeffekt . . . . . . . . . . . . . . . . . 395

17.5.2 Multiple Entstehungsgründe für Religion . . . . . . . . . . . . . . . . 397

17.6 Gefahren der Religion: Aufklärung, Religion und

Metaaufklärung . . . . . . . . . . . . . . . . . . . 399

17.6.1 Fundamentalismus . . . . . . . . . . . . . . . . . . . . . . 400

17.6.2 Aufklärung. . . . . . . . . . . . . . . . . . . . . 402

17.6.3 Metaaufklärung und Schlussplädoyer _ . . . . . . . . . . . . . . 403

Literatur . . . . . . . . . . . . . . . . . . . . . 405

Personenregister. . . . . . . . . . . . . . . . . 4421

Sachregister. . . . . . . . . . . . . . . . . . . . 429 


\section{Vorwort und Einleitung}

Die von Darwin begründete Evolutionstheorie hatte lange Zeit große Schwierigkeiten, nicht missverstanden zu werden und allgemeine Anerkennung zu finden. $\mathrm{Zu}$ ihrer Zeit stieß sie auf heftigen Widerstand. Immerhin lehrte sie, dass alles Leben auf der Erde bis hin zum Menschen in vielen Jahrmillionen durch natürliche Anpassungsprozesse entstanden sei, ohne dass dabei eine höhere zielgerichtete Schöpfungskraft, eine „lenkende Hand“ im Spiel war. Dadurch geriet die Darwin’sche Evolutionstheorie nicht nur mit Kirche und Religion, sondern auch mit alläglichen Vorstellungen des Common Sense in heftigen Konflikt.

Mittlerweile hat die biologische Evolutionstheorie eine kaum zu überbietende wissenschaftliche Erfolgsgeschichte aufzuweisen. Zumindest in der akademischen Fachwelt bildet sie die unbezweifelte Grundlage der Lebenswissenschaften. Gerade in den letzten beiden Jahrzehnten war eine stetig anwachsende Flut von Berichterstattungen über die Evolution des Lebens zu konstatieren, die mithilfe der neuen computergestützten Visualisierungsmethoden auch die visuellen Medien erobert hat. Zwar tobt die ideologische Auseinandersetzung zwischen Evolutionstheorie und religiös-kreationistischer Welterklärung nach wie vor, doch wird heute längst nicht mehr darüber verhandelt, ob die Evolutionstheorie eine akademisch akzeptable Theorie ist oder nicht. Es geht vielmehr umgekehrt um die Frage, ob eine rein naturalistische Welterklärung durch natürliche Evolutionsprinzipien ausreicht oder ob nicht doch, zumindest auf irgendeiner Ebene, auf intelligente Schöpfungskräfte zurückgegriffen werden muss, so wie dies in jüngster Zeit von Vertretern des anthropischen Prinzips in der Kosmologie vorgeschlagen wurde.

Wenn wir uns also in der Zeit einer stetig anwachsenden Flut von Literatur über Evolution befinden, warum dann ein weiteres Buch über Evolutionstheorie aus der Hand eines Wissenschaftsphilosophen? Weil sich in der Zwischenzeit, über die biologische Evolutionstheorie hinaus, ein wesentlich umfassenderes wissenschaftliches Theoriegebilde bzw. Paradigma herausgebildet hat, zu dem es bislang kaum eine Gesamtdarstellung gibt. Ich nenne dieses Paradigma bzw. Theoriegebilde die verallgemeinerte Evolutionstheorie. In einigen Fachgebieten wurde teilweise unabhängig voneinander die Darwin'sche Evolutionstheorie nämlich derart verallgemeinert, dass sie auf ganz neuartige Gebiete angewandt werden konnte, die außerhalb des Bereichs der Biologie liegen. Dabei werden die drei Darwin'schen Prinzipien der Reproduktion, Variation und Selektion von ihren biologisch-genetischen Grundlagen abgelöst und als abstrakte Eigenschaften dynamischer Systeme aufgefasst. 
Das Neuartige des vorliegenden Werkes soll darin liegen, diese verallgemeinerte Evolutionstheorie in interdisziplinärer und philosophischer Gesamtsicht dem Leser nahezubringen. Die biologische Evolution auf dem neuesten Wissensstand darf in diesem Werk selbstverständlich nicht fehlen, ebenso wenig wie die aktuelle philosophische Auseinandersetzung mit dem Kreationismus und dem anthropischen Prinzip. Doch dies bildet nur einen Teil einer Gesamtdarstellung, in der auch die kulturelle Evolutionstheorie und die evolutionäre Spieltheorie, die evolutionäre Erkenntnistheorie und Psychologie sowie die evolutionäre Ethik ihren Platz haben, bis hin zu neuen Theorien der präbiologisch-kosmischen Protoevolution, zur mathematischen Evolutionstheorie und zur wissenschaftstheoretischen Fundierung der abstrakten Theorie evolutionärer Systeme.

Mit etwas Wagemut kann die wissenschaftsphilosophische These formuliert werden, dass die verallgemeinerte Evolutionstheorie derzeit im Begriff ist, sich über ein Leitparadigma der Lebenswissenschaften hinaus zu einem übergreifenden Rahmenparadigma aller gegenstandsbezogenen Wissenschaften und der zugehörigen Wissenschaftsphilosophien zu entwickeln, von der Kosmologie bis zu den Kulturwissenschaften. Dies schließt nicht aus, dass daneben auch noch andere Leitparadigmen, beispielsweise die Systemtheorie oder die Handlungstheorie, eine bedeutende Rolle spielen können. Wir werden an entsprechenden Stellen dieses Buches herausarbeiten, wie solche Paradigmen in die verallgemeinerte Evolutionstheorie eingebettet werden können.

Zwar gab es schon Ende des 19. Jahrhunderts, noch zu Lebzeiten Darwins, Verallgemeinerungsversuche der Evolutionstheorie, jedoch in Gestalt des sogenannten Sozialdarwinismus, der auf mehreren gravierenden Fehlinterpretationen der Darwin'schen Evolutionstheorie beruhte. Beispielsweise gibt es in der modernen Evolutionstheorie keinen Automatismus zur Höherentwicklung, wie es der Sozialdarwinismus lehrte. Schon gar nicht gibt es ein Gesetz der Selektion des Stärksten, sondern nur eine Selektion des Bestangepassten, welche auch die Evolution von Altruismus und Kooperation befördern kann. Aufgrund solcher ideologischer Missdeutungen waren Verallgemeinerungsversuche der Evolutionstheorie bis in die frühen 1970er Jahre im geisteswissenschaftlichen Lager schlecht angesehen, was sich auch in der überwiegenden Ablehnung der Wilson'schen Soziobiologie seitens sozialund geisteswissenschaftlicher Disziplinen äußerte. Die bahnbrechenden Erfolge der Evolutionstheorie, von der "modernen Synthese“ (modern synthesis) bis zur Entdeckung des genetischen Codes, haben sich bis in die 1970er Jahre vorwiegend auf die Naturwissenschaften beschränkt. Dort führten sie jedoch durch zahlreiche neue Erkenntnisse, z. B. über die Existenz von Zufallsdriften und anderen nicht adaptionistischen Prozessen, zu einem wesentlich fortgeschritteneren Bild von biologischer Evolution, verglichen zu früheren Vorstellungen von biologischer Arterhaltung oder universalem Anpassungsdrang.

Die entscheidenden wissenschaftlichen Anstöße zur Entwicklung einer verallgemeinerten Evolutionstheorie erfolgten dagegen erst ab den späten 1970er Jahren. In diesem Zeitraum entwickelte Richard Dawkins den Begriff des Mems als kulturellem Gegenstück des Gens. Meme sind nicht angeborene, sondern erworbene menschliche Ideen und Fertigkeiten, die durch den Mechanismus der kulturellen Tradition reproduziert werden. Dawkins postulierte damit eine eigenständige Ebene der kul- 
turellen Evolution, und diese Idee wurde seither von vielen bekannten Wissenschaftlern weiterentwickelt. Der Begriff der Kultur wird dabei im weiten Sinne verstanden - er umfasst nicht nur Religion, Moral und Kunst, sondern auch Rechtssysteme und soziale Institutionen sowie insbesondere Sprache, Wissen und Technologie.

In etwa dieselbe Zeit fällt die Entwicklung der evolutionären Spieltheorie durch John Maynard Smith, die sich in der Ökonomie und den Sozialwissenschaften mittlerweile zum bedeutendsten Theorienansatz weiterentwickelt hat, mit dem die Evolution von Handlungsinteraktionen beschreibbar und durch Computersimulationen voraussagbar ist. Ebenfalls in den 1970er Jahren hat Neil A. Campbell seine auf Begriffen der Variation, Selektion und Retention basierende verallgemeinerte Evolutionstheorie entwickelt, die auch präbiologische Prozesse in der Chemie oder im Kosmos erfasst und sich ebenfalls zwanglos in die hier entwickelte Konzeption der verallgemeinerten Evolutionstheorie einfügt.

Campbell hat auch wesentlich zur evolutionären Erkenntnistheorie beigetragen, die schon in den 1940er Jahren durch Lorenz angeregt wurde. Die ältere evolutionäre Erkenntnistheorie steht mit ihrem Glauben an den evolutionären Wahrheitsfortschritt in interessantem Kontrast zur jüngeren Disziplin der evolutionären Psychologie, insbesondere der evolutionären Kognitionspsychologie. Für die Analyse dieses Gegensatzes erweist sich der Rahmen der verallgemeinerten Evolutionstheorie als ebenso nützlich wie für die Analyse zweier weiterer Gebiete, die den Abschluss dieses Buches bilden. Das erste Gebiet bildet die Frage nach den Möglichkeiten der Evolution von sozialer Kooperation, einer der Hauptfragen der evolutionären Spieltheorie, zu der es mittlerweile eine Flut von Spezialliteratur gibt. Soziale Kooperation kann zwar vergleichsweise leicht entstehen, doch ihre nachhaltige evolutionäre Stabilisierung gegenüber „Ausbeutern“ sieht sich bekanntlich hartnäckigen Schwierigkeiten gegenüber und erfordert komplexe soziale Mechanismen wie z. B. gemeinschaftliche Reputations- oder Sanktionssysteme. Das zweite Gebiet ist die Erklärung der evolutionären Nachhaltigkeit von Religionen, auch noch in den zivilisatorisch fortgeschrittensten Teilen dieser Welt. Als Erklärung dieses Phänomens wird der verallgemeinerte Placeboeffekt vorgeschlagen. Auch dieser Effekt lässt sich nur im Rahmen der verallgemeinerten Evolutionstheorie befriedigend erfassen.

Fasst man die gesamte Literatur in jenen Disziplinen ins Auge, die in das so umschriebene Gebiet der verallgemeinerten Evolutionstheorie fallen, so erreicht diese Literatur einen hohen Grad an Interdisziplinarität und eine für Laien wie Spezialisten verwirrende Komplexität. Im vorliegenden Buch wird diese Komplexität systematisiert, sprachlich vereinheitlicht und allgemein verständlich dargestellt, ohne besondere Vorkenntnisse vorauszusetzen. Das besondere Augenmerk gilt dabei den philosophischen Grundlagen und Letztfragen, die immer wieder angesprochen werden.

Als weitere Besonderheit dieses Buches werden wir aufzeigen, wie sich die Beschreibungsmodelle der biologischen Populationsgenetik, der Memtheorie und der evolutionären Spieltheorie auf Variationen ein und desselben Grundmodells zurückführen lassen. Durch all ihre vielfältigen und sehr konkreten Anwendungen hindurch wird nämlich die Gesamtdisziplin der verallgemeinerten Evolutionstheorie in ihrem Kern von einer einheitlichen und mathematisch im Detail ausformulierten 
Theorie zusammengehalten, und diese erst rechtfertigt es, von der verallgemeinerten Evolutionstheorie nicht nur in einem metaphorischen, sondern genuin theoretischen Sinn zu sprechen.

Der besseren Orientierung halber ist das Buch in fünf aufeinander aufbauende Teile gegliedert, die Kapitel sind jedoch fortlaufend durchnummeriert. Abbildungen, Tabellen und Boxen (die allgemein-philosophische Erläuterungen enthalten) sind kapitelweise nummeriert (z. B. Abb. $3.4=$ Abb. 4 von Kap. 3). Kapitel- und Abschnittsverweise mit Literaturbezug beziehen sich auf das genannte Buch (z. B. Meier 2000, Kap. 5) und mit Verweispfeilen auf das vorliegende Buch ( $\triangleright$ Kap. 5).

Teil I behandelt die historische Entwicklung und die modernen Grundlagen der biologischen Evolutionstheorie sowie aktuelle Theorien zu protoevolutionären Entwicklungsprozessen im Kosmos. Dabei konzentrieren wir uns auf die philosophische Auseinandersetzung mit dem Kreationismus, dem anthropischen Prinzip und den letzten Warum-Fragen. In Teil II werden die grundsätzlichen Schritte zur Verallgemeinerung der Evolutionstheorie vorgenommen sowie die wissenschaftstheoretischen Grundfragen und ethischen Konsequenzen der verallgemeinerten Evolutionstheorie behandelt. Bei den wissenschaftstheoretischen Grundfragen geht es insbesondere um das moderne Verständnis des Begriffs der Funktion und um die Frage der Reduzierbarkeit bzw. Nichtreduzierbarkeit von „höheren“ Wissenschaften wie z. B. der Biologie auf die Physik. Das ethische Schlusskapitel enthält eine Kritik des Sozialdarwinismus sowie eine Diskussion von Prinzipien einer evolutionären Ethik und ihrer Beziehung zu Prinzipien der humanistischen Ethik.

Teil III enthält die Übertragung der verallgemeinerten Evolutionstheorie auf die kulturelle Evolution. Nach einer kritischen Einführung in die aktuelle Kontroverse um die Theorie der Meme werden aktuelle Anwendungen der kulturellen Evolutionstheorie vorgestellt sowie die Wechselwirkungen zwischen biologischer, kultureller und individueller Evolution analysiert. Daran anknüpfend werden in Teil IV die mathematischen Modelle der Populationsgenetik und der Populationsdynamik von Memen in vereinheitlichter Weise dargestellt und nahtlos in die Modelle der evolutionären Spieltheorie übergeführt. Dieser Teil ist aus zwei Gründen fundamental. Erstens, weil in ihm das einheitliche Kernstück der verallgemeinerten Evolutionstheorie herausgearbeitet wird. Zweitens, weil darin gezeigt wird, warum bedeutende evolutionstheoretische Einsichten erst durch die präzisen Modelle der Populationsdynamik und ihrer mathematisch-computergestützten Analyse ermöglicht werden. Zugleich ist Teil IV der einzige der fünf Teile, welcher dem Leser etwas „Gedankenakrobatik" in Form mathematischer Gleichungen vom Niveau gymnasialer Oberstufenmathematik zumutet, deren wesentlicher Gehalt aufgrund der grafischen Illustrationen, aber auch ohne genaues mathematisches Verständnis nachvollzogen werden kann. Teil $\mathbf{V}$ beschließt das Buch schließlich mit einer vertieften Analyse des Problems der Evolution von sozialer Kooperation sowie der Evolution von menschlicher Kognition, Weltanschauung und Religion im Spannungsfeld zwischen Rationalität und Irrationalität.

Für wertvolle Hilfestellungen bei der Erstellung und Abfassung des Buchmanuskripts danke ich insbesondere meinem Kollegen Axel Bühler, meinen Mitarbeitern Katja Ludwig, Erasmus Scheuer, Hakan Beseoglu und Veronika Linke, meiner Frau Dorothea Schurz-Weisheit und meiner Tochter Christine Schurz, sowie meinen 
geschätzten Düsseldorfer Diskussionspartnern und Kollegen Dieter Birnbacher, Werner Kunz, Hans Geisler, Klaus Lunau, Michael Baurmann, Markus Werning, Ioannis Votsis, Ludwig Fahrbach, Eckhart Arnold, Elmar Hermann, Jens Fleischhauer und Matthias Unterhuber. Dank für hilfreiche Korrespondenz schulde ich ferner Gerhard Vollmer, Wolfgang Wickler, Lucie Salwiczek, Marcel Weber, Martin Carrier, Paul Hoyningen-Huene, Hannes Leitgeb, Hartmut Kliemt, Heinz Wimmer, Josef Perner, Gernot Kleiter, Niki Pfeifer, Ruth G. Millikan, Samir Okasha, Philip Kitcher, Brian Skyrms, Alvin Goldman, Simon Huttegger, Reiner Hegselmann, Igor Douven und Kevin Kelly. Für die gute Zusammenarbeit mit dem Verlag bedanke ich mich bei Andreas Rüdinger und Heidemarie Wolter. Mich selbst hat das Gebiet der verallgemeinerten Evolutionstheorie im Laufe meiner Arbeit immer mehr fasziniert, und ich wünsche mir, dass etwas von dieser Faszination auf die geschätzte Leserin bzw. den geschätzten Leser übergeht.

Düsseldorf, im März 2010

Gerhard Schurz 